\title{
Chapter 15 \\ Twin-Control Evaluation in Industrial Environment: Aerospace Use Case
}

\author{
Mikel Armendia, Mani Ghassempouri, Guillermo Gil, \\ Carlos Mozas, Jose A. Sanchez, Frédéric Cugnon, \\ Luke Berglind, Flavien Peysson and Tobias Fuertjes
}

\subsection{Introduction}

Within Twin-Control project several features have been developed and validated at research level, showing promising results, as presented in the previous chapters of the book. However, since its gestation, Twin-Control project has aimed to provide

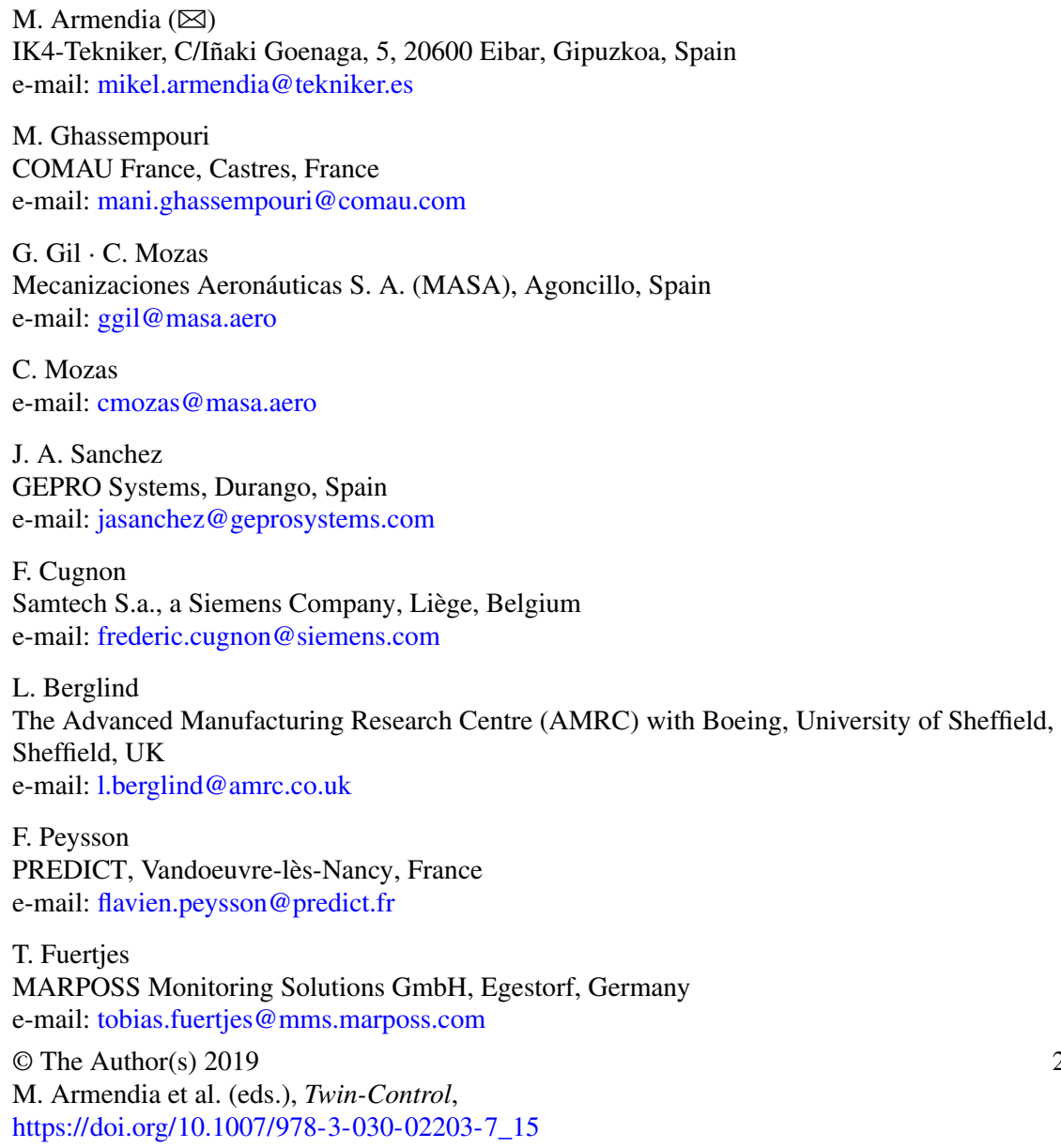


the required industrial validation. In this line, two validation use cases are presented in the project, from two of the more demanding sectors in European industry and with very different requirements: aerospace and automotive. This chapter presents the results obtained in the application of Twin-Control in the aerospace use case.

Section 15.2 of this chapter provides a summary of the validation use case. In Sect. 15.3, the applied industrial evaluation approach is presented. Section 15.4 deals with the implementation activities and the obtained results, including the impact caused by these features in the end-users. Finally, the last section covers the conclusions.

\subsection{Use Case Description}

The aerospace validation scenario is located at Mecanizaciones Aeronáuticas S.A. (MASA) plant in Agoncillo, near Logroño in Spain. For the evaluation, GEPRO 502, 512 and 304 machine tools are used. The architecture of the three machines is similar, but they differ in the number of axes, dynamic capabilities, spindle type, etc. Even of the three machines have been monitored, the GEPRO 502, depicted in Fig. 15.1, has been used as the main reference to implement Twin-Control features.

For the aerospace use case, a new machining process, illustrated in Fig. 15.2, has been used. This process has been defined for the project and combines features of different test pieces usually applied by MASA for internal validations. The part is a

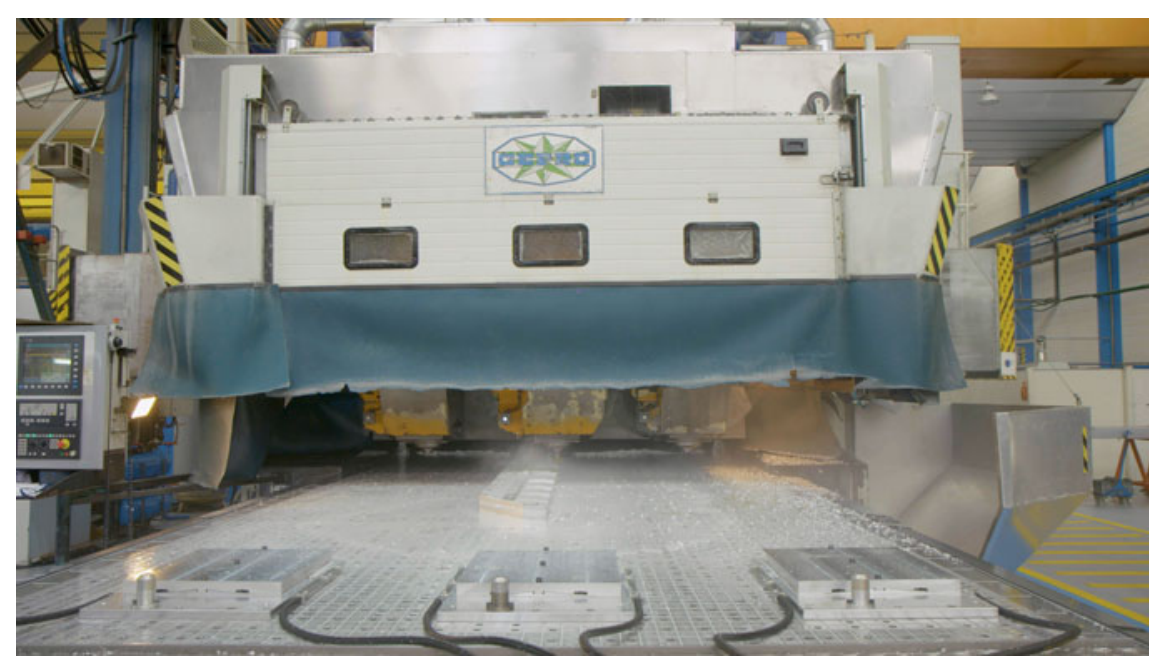

Fig. 15.1 General view of the GEPRO 502 machine, available in MASA installations, used in the aerospace validation scenario 


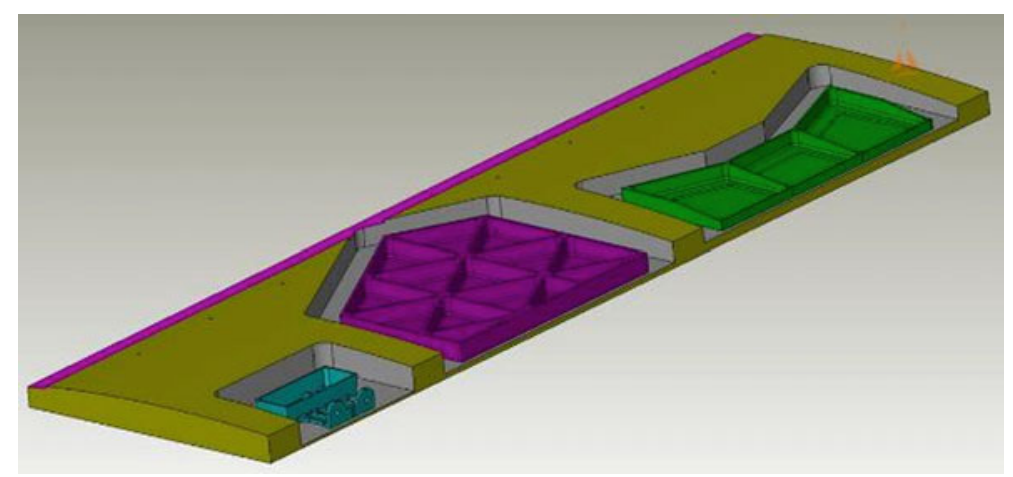

Fig. 15.2 Part used in the aerospace validation use case

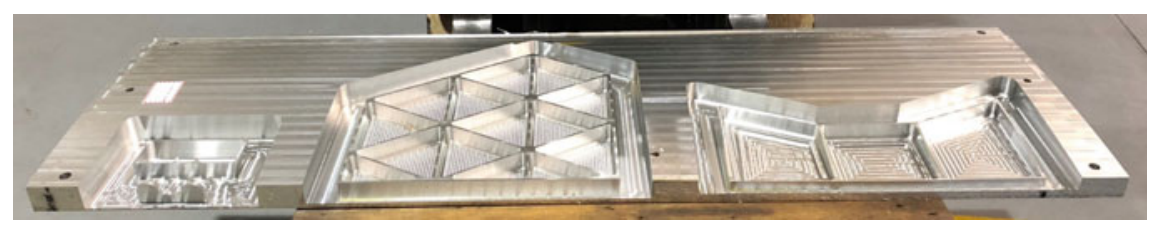

Fig. 15.3 Validation part machined at MASA

large plate of aluminium that is manufactured using 3- and 5-axis milling, as well as drilling operations. Its dimensions are $2000 \times 600 \times 65 \mathrm{~mm}$.

The evaluation is mainly focused in the triangular pocket region in the middle, represented by purple region in Fig. 15.2. This geometry requires movements aligned in the $X$ - and $Y$-axes, as well as interpolated movements of these two axes. Through this test the machine tool precision in the linear axis, $X$ and $Y$, can be evaluated, both when they work individually and interpolated. During the corner machining of the triangular pockets, the machine performs feed rate, acceleration, cutting direction and chip thickness that allow evaluating the dynamic performance of the machine (speed, acceleration and jerk) and the quality of this type of operation.

The validation part has been machined several times (Fig. 15.3) to acquire data through the monitoring system and use it for the validation of the different TwinControl features. The machined validation part is shown in Fig. 15.3.

\subsection{Evaluation Strategy}

The evaluation strategy will be linked to the different scenarios of use (SOU) defined by the aerospace end-users at the beginning of the project: 
- Scenario of Use 1: Machine tool design

- Scenario of Use 2: Process design

- Scenario of Use 3: In-line operation

- Scenario of Use 4: Maintenance

For each scenario of use, results obtained with Twin-Control are presented, and the impact on end-users is evaluated.

\subsection{Scenario of Use 1: Machine Tool Design}

The tools and capabilities developed in Twin-Control create a new design environment for machine tool builders which allow to optimize the machine design through simulations. Since in aerospace, process performance overcomes energy efficiency in importance, and the work has been focused in the first feature.

\subsubsection{Virtual Machine Tool with Integrated Process Models}

\subsubsection{Implementation and Results}

A FEM-based kinematic and dynamic model of the GEPRO 502 machine has been done using SAMCEF Mecano software. The model, represented in Fig. 15.4, covers machine structure, feed drives and integrates control. Specific validation of this simulation model was performed by using hammer tests and positioning movements. The results are presented in Sect. 2.1 of this book.

The machining module has been configured for the three machines of the aerospace use case. For that, characteristics of the cutting tools applied in the validation use case have been collected and stored in a table shape database, reproduced in Table 15. 1, to be used by the simulation tool.

The integrated simulation tool developed in the Twin-Control project is validated by comparing simulated data with data measured during the real process shown in Fig. 15.3.

Once the CAD model of the validation workpiece, shown in Fig. 15.2, is positioned properly on the CAD model of the machine, it is translated to STL format in order to be used in the VMT model depicted in Fig. 15.5.

Validation consists of simulating four machining sequences as shown in Fig. 15.6: a roughing operation of the external groove (blue path), a roughing operation of one triangular pocket (red path), a finishing operation of the vertex of the pocket (black path) and finishing operation of the walls of the pocket (green path). Position target functions replicated from the ISO code executed in the machine are used to feed the Virtual Machine Tool model. 


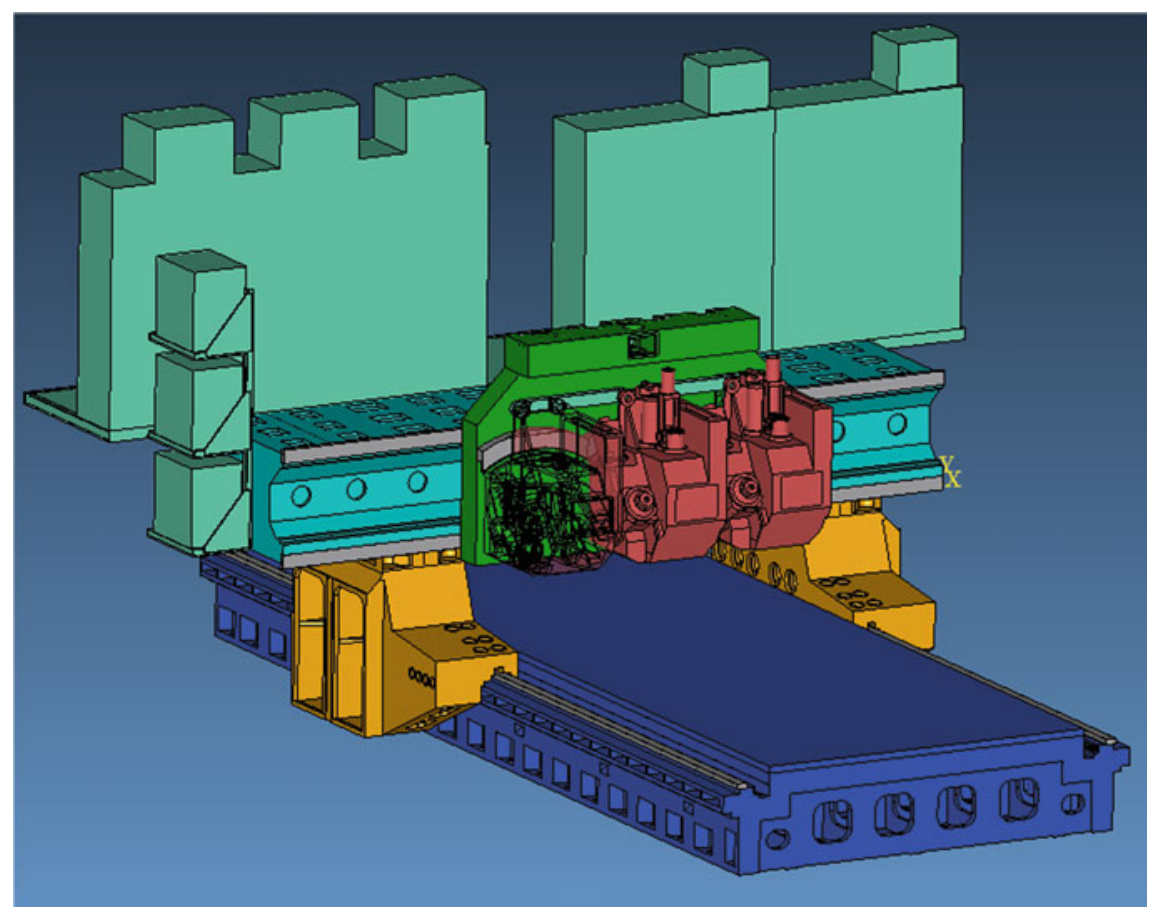

Fig. 15.4 CAD model of the GEPRO 502 machine used for the its Virtual Machine Tool model

The blue machining sequence in Fig. 15.6a, defined as external groove, can be decomposed in 9 equivalent trajectories at different heights defined by the axial depth

Table 15.1 Characteristics of the tools needed in aerospace use case

\begin{tabular}{l|l|l|l|l|l|l|l}
\hline Tool \# & D & Rc & Beta & Lam & Nflut & ToolDir & Zmax \\
\hline 1 & 50 & 4 & 0 & 0.610865 & 3 & 0 & 15 \\
\hline 2 & 25 & 4 & 0 & 0.610865 & 3 & 0 & 30 \\
\hline 3 & 5 & - & 0 & 0.610865 & 2 & 0 & 20 \\
\hline 4 & 40 & 4 & 0 & 0.610865 & 3 & 0 & 15 \\
\hline 5 & 25 & 5 & 0 & 0.610865 & 3 & 0 & 30 \\
\hline 6 & 20 & 5 & 0 & 0.610865 & 3 & 0 & 20 \\
\hline 7 & 20 & 0 & 0 & 0.610865 & 3 & 0 & 20 \\
\hline 9 & 20 & 4 & 0 & 0.610865 & 3 & 0 & 20 \\
\hline 10 & 16 & 4 & 0 & 0.610865 & 3 & 0 & 20 \\
\hline
\end{tabular}

$D$ Tool Diameter [mm]; $R c$ nose radius of tool [mm]; Beta taper angle of tool [rad]; Lam Helix angle [rad]; Nflut Number of flutes [-]; ToolDir cut rotation direction [-]; Zmax max Z value for virtual tool $[\mathrm{mm}])$ 


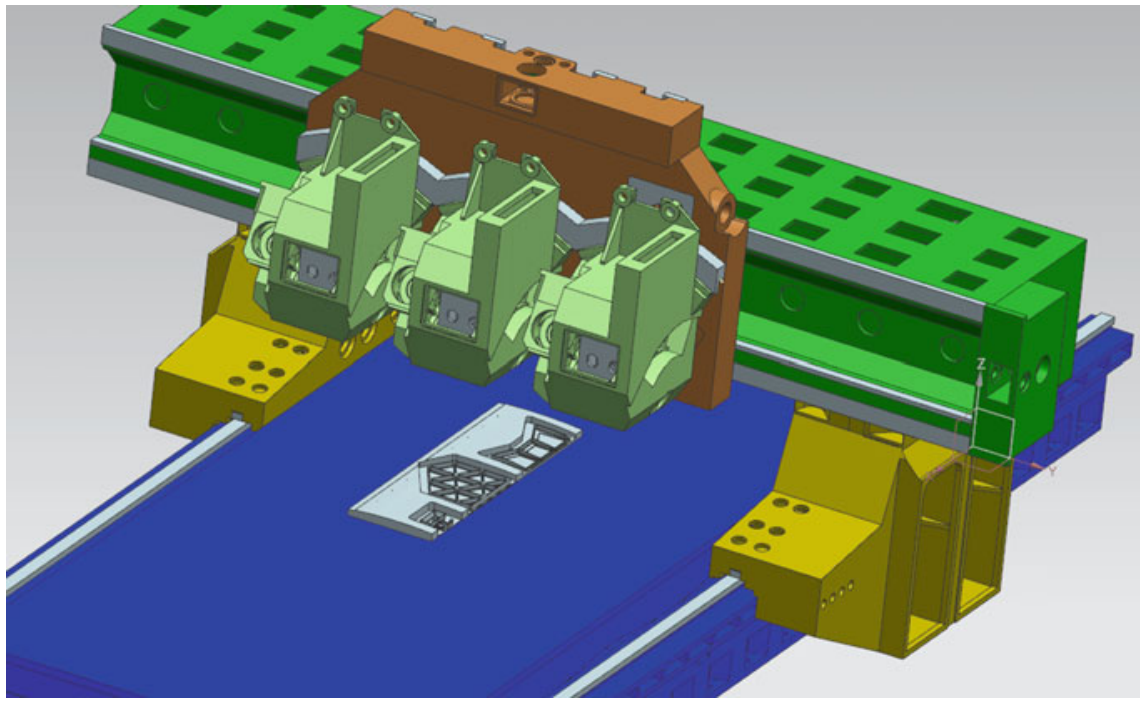

Fig. 15.5 CAD model of the workpiece positioned on the machine model
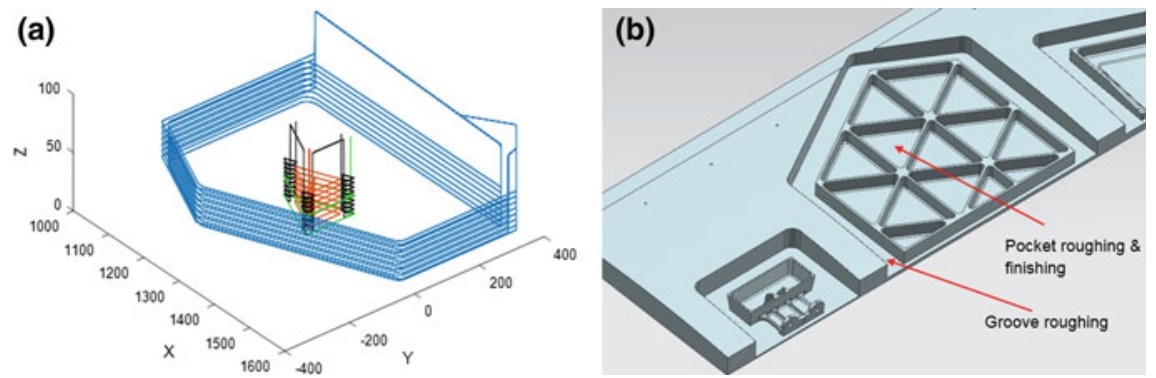

Fig. 15.6 Machining sequences for the validation of machining performance in the aerospace validation scenario

of cut of $5 \mathrm{~mm}$. For each pass, the workpiece STL file generated from the previous one is used, replicating the real machining procedure. In Fig. 15.7, the evolution of the groove machining using the simulation tool is presented graphically.

After the external groove, the triangular pocket roughing is simulated, corresponding to the red machining sequence of Fig. 15.6a. Again, the roughing process is separated in different phases which are simulated taking into account the workpiece geometry of the previous pass. After the roughing operation, triangular pockets are finished by applying two steps. The first one consists in the corner finishing and corresponds to the black machining sequence of Fig. 15.6a. Each vertex is machined by performing three passes at different heights. The second finishing step consists in the wall finishing of the triangle pockets and corresponds to the green machining 

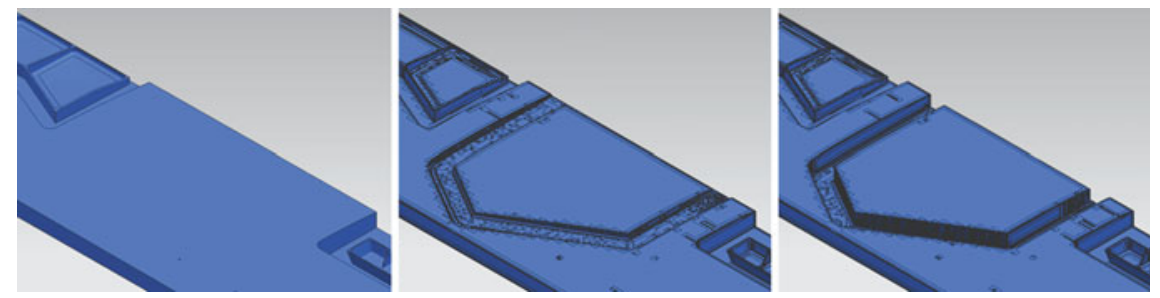

Fig. 15.7 CAD (STL) evolution during the simulation of the external groove machining in the aerospace use case
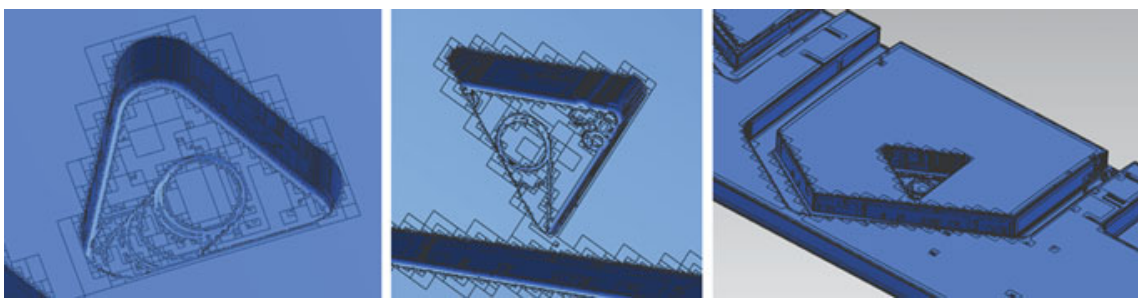

Fig. 15.8 Triangular pocket machining simulation sequence (from left to right): roughing, corner finishing and wall finishing

sequence of Fig. 15.6a. In this case, two passes at different heights are performed. Figure 15.8 presents the complete sequence to machine the triangular pockets.

Figures 15.9 and 15.10 show the simulation results for the pocket roughing and wall finishing operations, respectively, comparing them to the monitored data during real machining at MASA. Although some errors can be observed for some steps of the process, surely due to tool-workpiece engagement estimation inaccuracy, the obtained results match quite well, specially, for roughing operations where higher forces are present.

\subsubsection{Impact}

GEPRO is not a general-purpose machine tool manufacturer. Each GEPRO machine is developed to machine a specific part or part family, always from aerospace sector. In some cases, due to the big size of the machines and the high costs, GEPRO works in the retrofitting of older machines to use the big structural components.

Due to small size of the company and the presented features, GEPRO does not fabricate prototypes for the new machine tool models. Indeed, as each machine is taken as a new development, all GEPRO machines can be taken as prototypes, but they must be completely functional in production.

The current product development stages reproduced in the top of Fig. 15.11, and the correspondent average duration, for GEPRO are: 


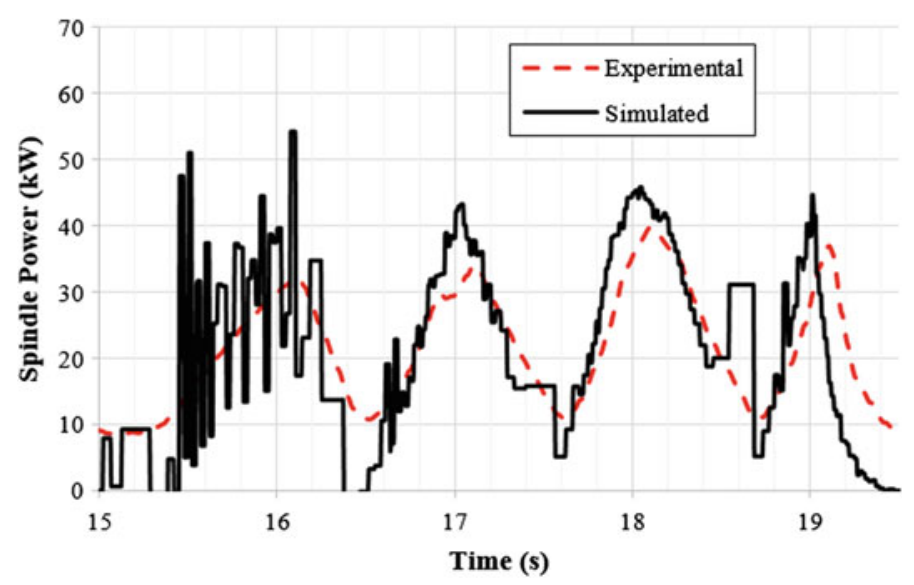

Fig. 15.9 Comparison of the simulation results obtained with the integrated simulation tool and real monitored data for the triangular pocket roughing operation

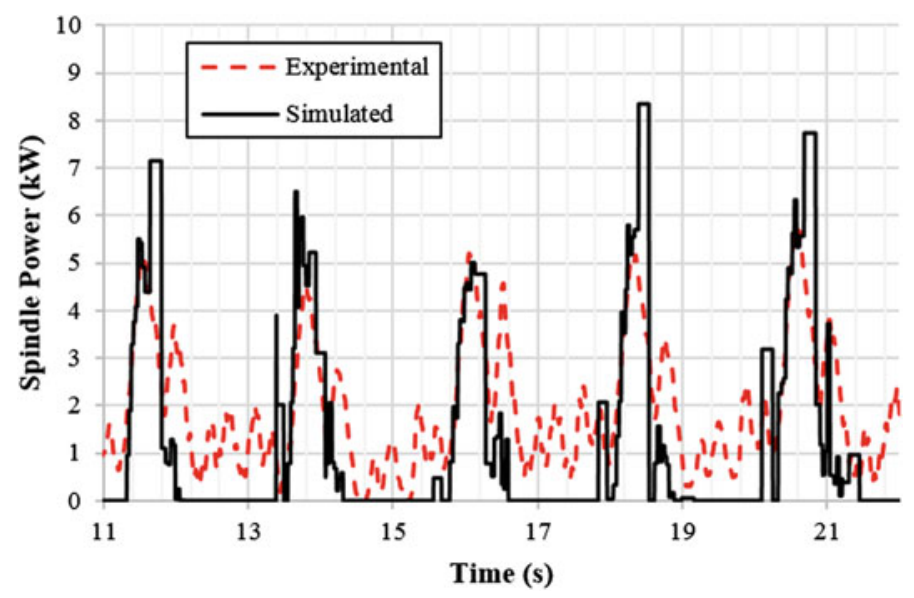

Fig. 15.10 Comparison of the simulation results obtained with the integrated simulation tool and real monitored data for the triangular corner finishing operation

- Conceptual design of the new machine (0.5 months)

- Detailed design (3-4 months)

- Design review and modifications (0.5 months)

- Component fabrication and purchasing (3.5 months)

- Assembly (2-3 months)

- Commissioning (2-3 months)

- Validation and optimization tests (0.5 months).

The application of Twin-Control in the product development of a company like GEPRO will have a direct effect in the commissioning stage, as shown in the bottom 
Typical Product Development (Current) $=12-15$ months

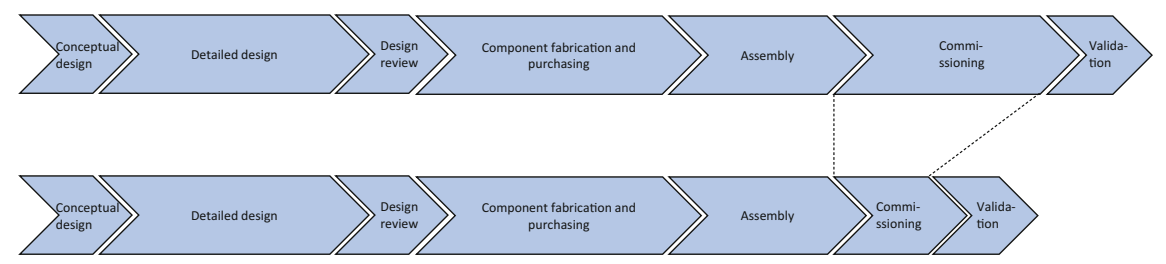

Fig. 15.11 Comparison of the current product development process for GEPRO and the proposed one with the application of Twin-Control

of Fig. 15.11. Indeed, if Twin-Control models are used in the design stage, the developed machine design will be optimized against the required manufacturing process. A reduction of a $10 \%$ in the total development time is expected. Contrary to COMAU case, this reduction affects all the machines manufactured.

\subsection{Scenario of Use 2: Process Design}

For this scenario of use, the integrated simulation tool and the process models developed in the project are of special interest. The possibility to simulate in advance the manufacturing processes provides a great chance to reduce design time and to define optimized processes, leading to a minimization of the set-up time and a better performance of the process.

\subsubsection{Virtual Machine Tool with Integrated Process Models}

\subsubsection{Implementation and Results}

The same implementation presented in scenario of use 1, machine tool design, is applied in this scenario. Apart from that, for the simulations carried out with the GUI containing the process models, additional hammer tests have been performed at the GEPRO machine to get the FRF of the tooltip with all the used tools Fig. 15.12 shows an example of FRF.

The collected machine data is applied directly in the process model's GUI, so that the simulated tool path follows the measured tool path. The resulting simulated spindle power is then compared with the measured spindle power. The simulated and measured spindle power data is shown for the external grooving, blue machining sequence in Fig. 15.6a with a previous facing operation, in Fig. 15.13.

A closer look at the external grooving operation shows that the measured data follows the same path as the measured data and that the results closely agree, especially 
(a)

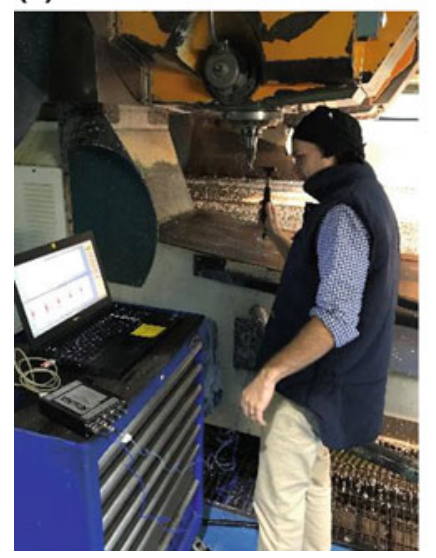

(b)
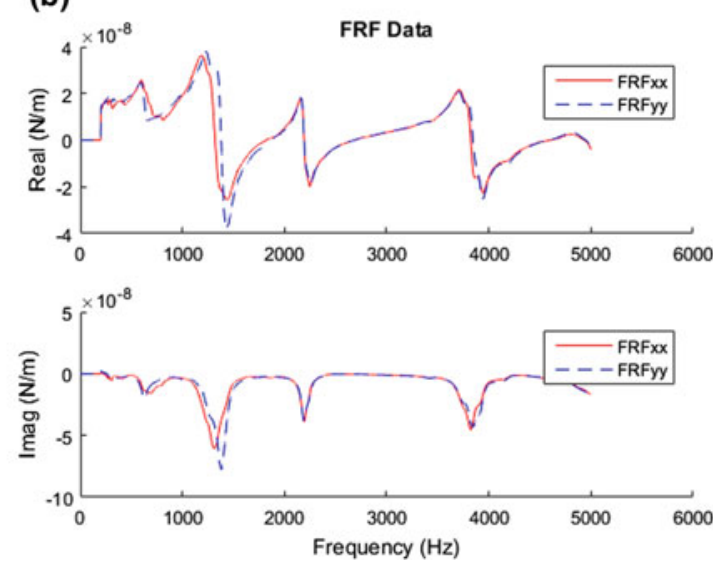

Fig. 15.12 Tool FRFs in the GEPRO 502: a Hammer tests. b Example of FRF for the tool \#1 (from Table 15.1)

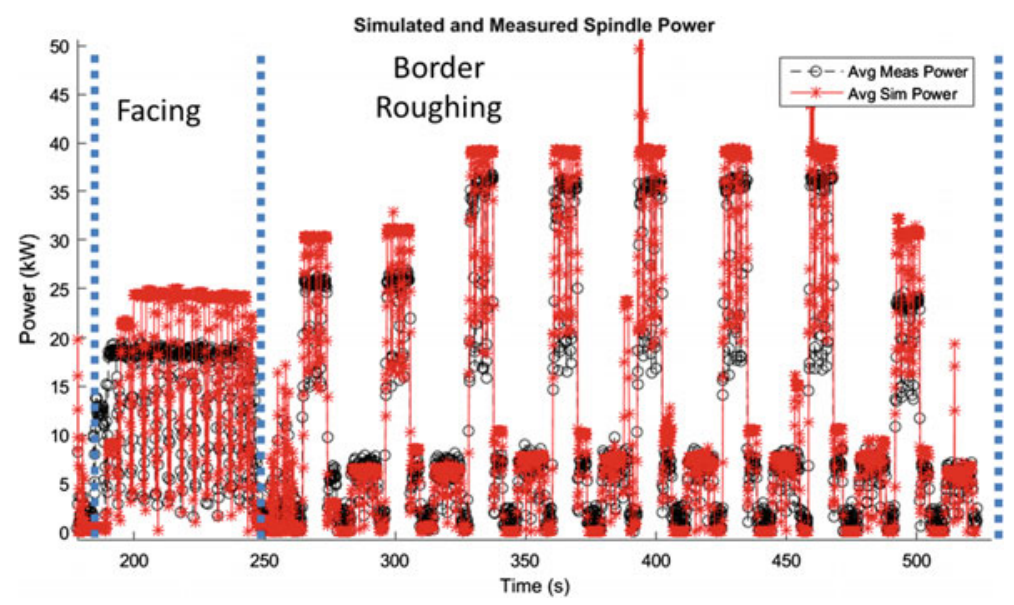

Fig. 15.13 External grooving operation of MASA process: simulated and measured spindle power

at the lower power levels. In Fig. 15.14, the different regions of the operation are identified, showing the amount of power required for each feature of the geometry.

The simulated and measured power results for the triangle pocketing are shown in Fig. 15.15. This operation is composed of a triangular roughing pass, a corner roughing pass and a finishing pass, and required two separate tools in the simulation. The results for the triangle feature also show close agreement between the simulated and measured power values, even at low power rates for the corner roughing and finishing passes. 


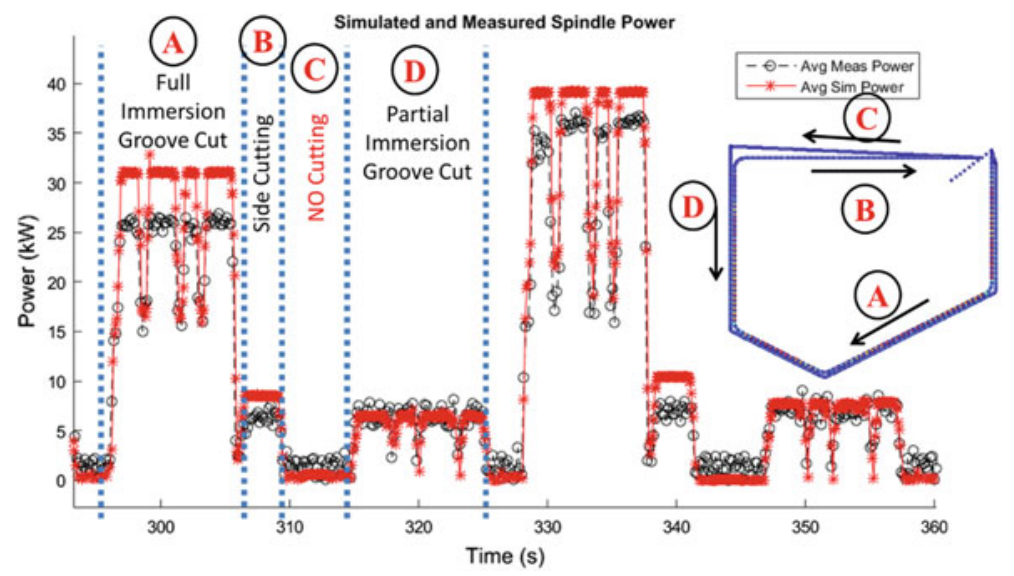

Fig. 15.14 External grooving operation of MASA process: detailed view of the simulated and measured spindle power for a single pass
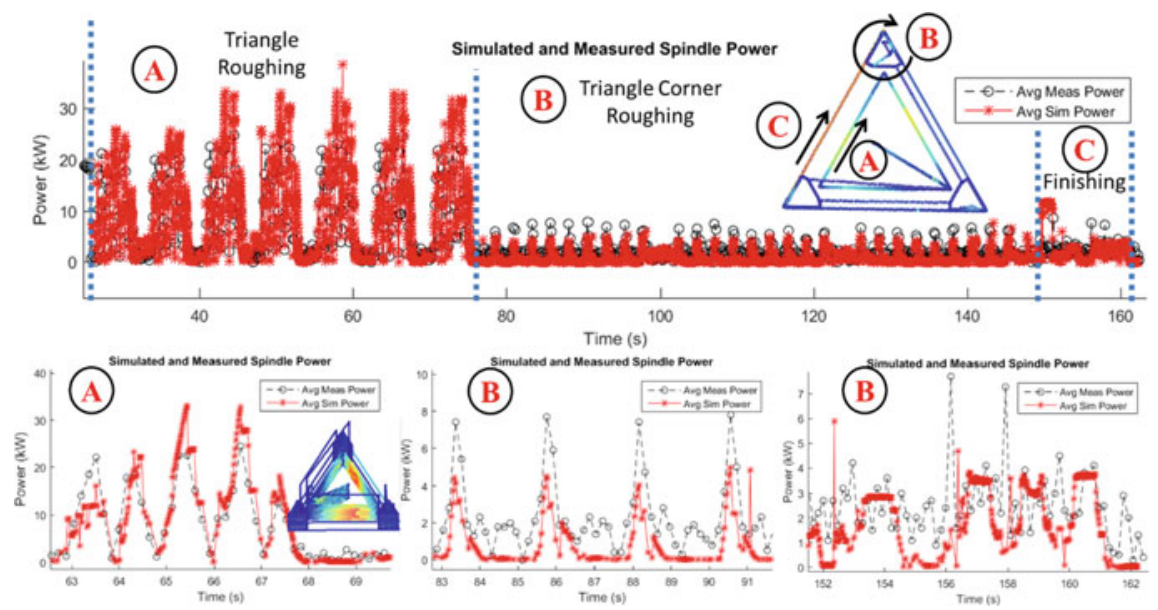

Fig. 15.15 Triangular pocket milling at MASA use case: simulated and measured spindle power

It can be concluded that the application including process models is a very useful tool for machine tool designers. Of course, it does not provide such a complete range of results as the integrated simulation model based on machine tool FEM, but simulations are faster to configure and run. Figure 15.16 shows a comparison of the results obtained for the triangular pocket roughing using the integrated simulation tool, the process model's GUI and the monitored data during real machining. It can be observed that both models' results are very similar and are close to experimental data.

A key aim of the Twin-Control concept is to improve process efficiency through integration of simulation and measurement. As a demonstration, the process simula- 


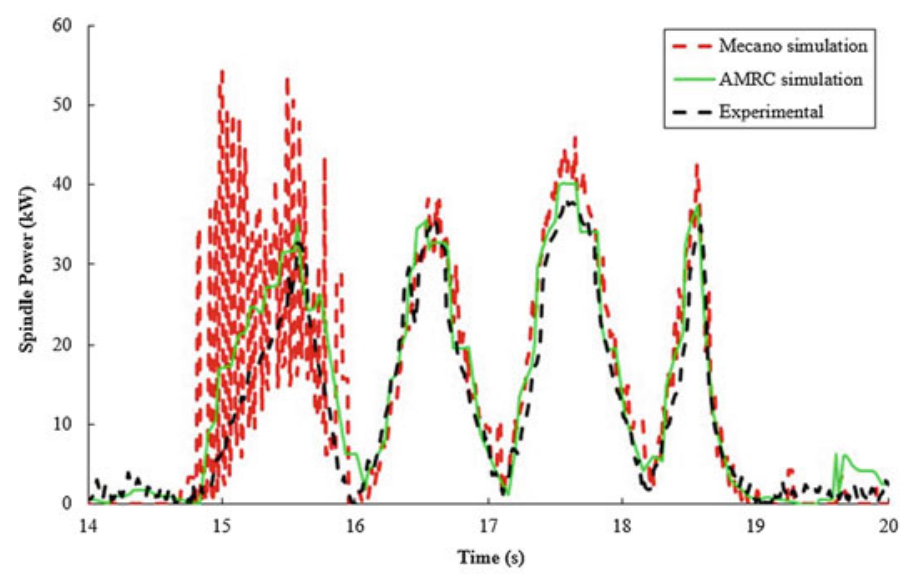

Fig. 15.16 Comparison of the results obtained with the integrated simulation tool (Mecano), process models (AMRC) and monitored values for

tion and measurements results from Fig. 15.15 have been applied to identify process improvements. Different strategies are tested on three triangular pockets, where each pocket is broken down into three operations (roughing, vertex finishing and wall finishing), and each operation is broken down, again, in three steps where machining conditions vary. A summary of the process parameters is provided in Table 15.2. For the pocket roughing operation (steps 5001-5003), the axial step is varied between 5 and $7 \mathrm{~mm}$, so the number of roughing passes can be reduced. For the corner roughing operation (steps 5004-5006), the feed rate is varied between 6750 and $8100 \mathrm{~mm} / \mathrm{min}$, and for the wall finishing operation (steps 5007-5009), the feed rate is varied between 6750 and $8100 \mathrm{~mm} / \mathrm{min}$.

Three new triangular pockets have been machined at MASA using the process parameters from Table 15.2 and test if the new parameters produce an acceptable part. During machining, measurements of the machine axes and spindle torque are again collected through the installed monitoring system. This data provides an opportunity to verify if the simulations results can capture subtle changes in the process. Figure 15.17 shows the simulated and measured spindle power results for the three triangle roughing operations. At this scale, the simulation results closely follow the measured power results as the depth of cut is increased from 5 to $7 \mathrm{~mm}$.

The results from Fig. 15.18 show the simulated and measured spindle torque for the vertex finishing operation, using three machining conditions. In this case, again, the simulation can predict small changes in power as the feed rate is increased, although the changes are smaller in this case.

The results from Fig. 15.19, that presents the comparison for the wall finishing operation, indicate that the measurement and simulation results track less accurately when the power or torque magnitudes are small.

The results from this second phase of trials at MASA have shown that the simulations are able to be used to improve new and existing processes. The ability to 


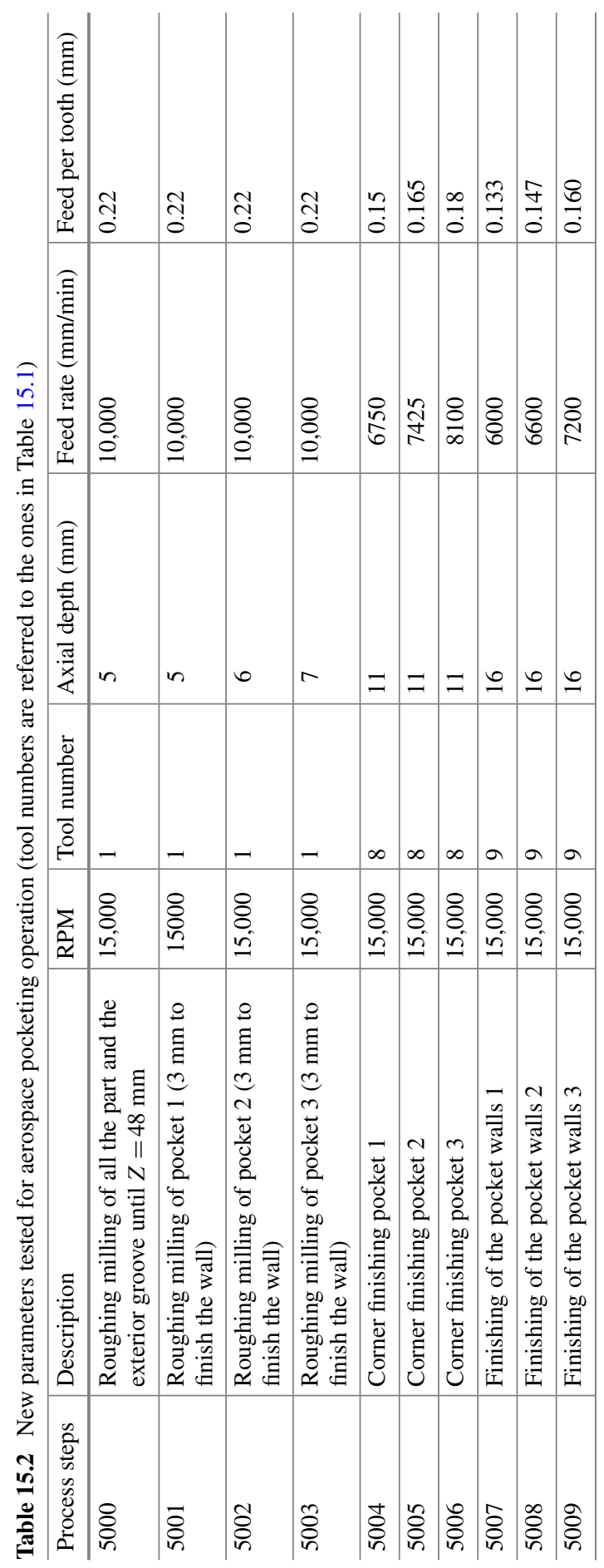


(a)

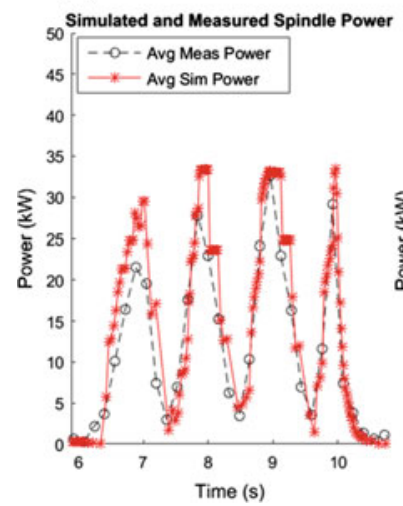

(b)

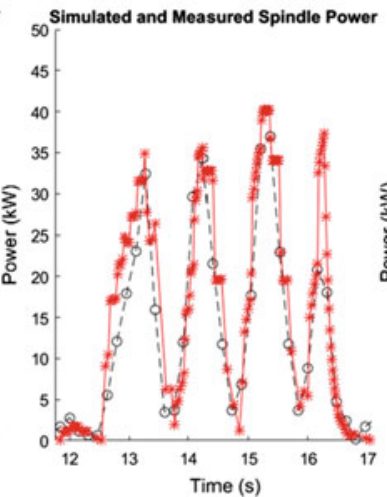

(c)

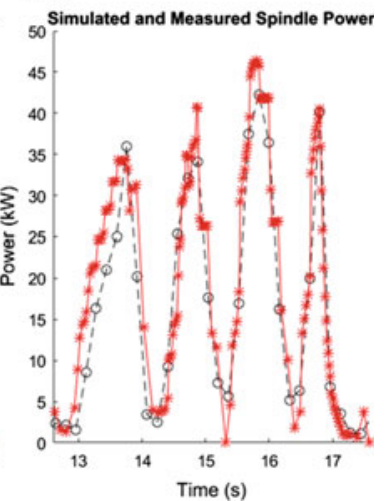

Fig. 15.17 Triangle roughing operation of a pocket 1 (operation 5001), b pocket 2 (operation 5002) and $\mathbf{c}$ pocket 3 (operation 5003)

(a)

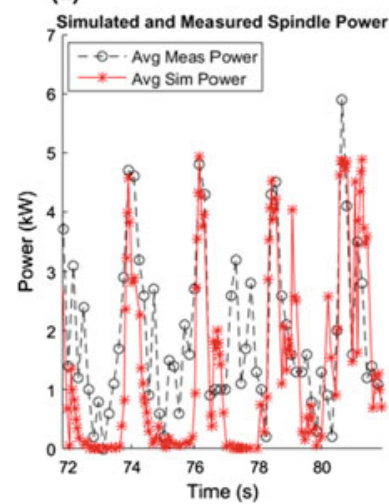

(b)

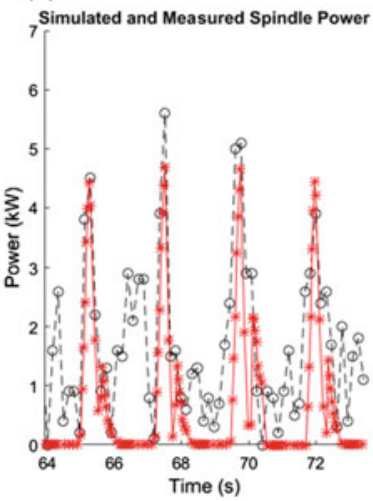

(c)

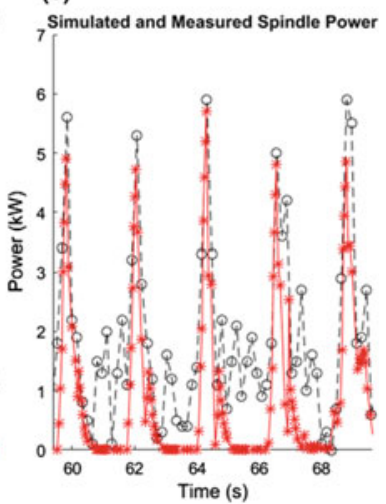

Fig. 15.18 Triangle vertex finishing operation of a pocket 1 (operation 5004), b pocket 2 (operation 5005) and $\mathbf{c}$ pocket 3 (operation 5006)

accurately simulate subtle changes to the process allows process planners to first identify potential areas of improvement and then be able to explore the effect each parameter will have on the process outcome. Ultimately, this process can be used to fully test new processes off machine and increase confidence in a process before the first part is produced.

\subsubsection{Impact}

Table 15.3 shows the phases to implement a new manufacturing process in MASA, from aerospace sector, including an estimation of the duration of each phase. Since 
(a)

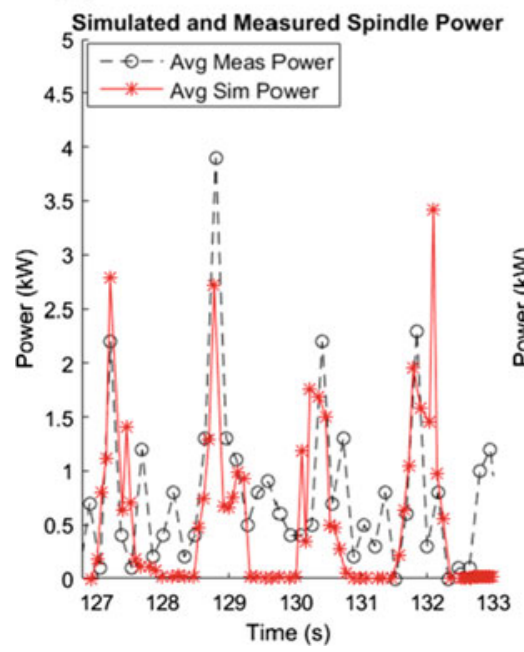

(b)

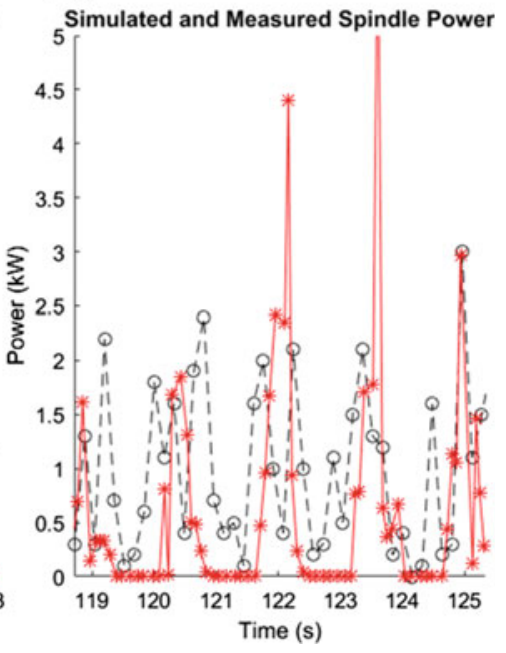

Fig. 15.19 Triangle wall finishing operation of a pocket 2 (operation 5008) and b pocket 3 (operation 5009)

Table 15.3 Average duration of the different stages of the new process design and set-up procedure for MASA (aerospace validation scenario) and the impact expected with the application of TwinControl features

\begin{tabular}{l|l|l|l}
\hline \multicolumn{2}{l|}{ Activities } & $\begin{array}{l}\text { Duration } \\
(\text { days })\end{array}$ & $\begin{array}{l}\text { Expected duration with } \\
\text { Twin-Control (days) }\end{array}$ \\
\hline 1 & Study of the geometry & 5 & 5 \\
\hline 2 & Process definition & 3 & 3 \\
\hline 3 & MT and tool choice & 2 & 1 \\
\hline 4 & CNC programming and tooling design & 21 & 21 \\
\hline 5 & Vericut simulation & 3 & 5 \\
\hline 6 & 1 st part machining & 1 & \\
\hline 7 & Inspection & 2 & \\
\hline 8 & Final modification in process & 6 & \\
\hline & Total average duration & 45 & 35 \\
\hline
\end{tabular}

small batches are usually required from customers, and hence, this implementation is performed with a high frequency.

In the third column, the impact expected by the application of Twin-Control process simulation capabilities is included. The biggest impact is expected in two stages:

- Machine Tool selection: MASA has several machines at its workshop, with very different configurations and characteristics. One of the most important steps for a successful process implementation is the selection of the best machine tool for 
the specific process. With the implementation of Twin-Control simulations, the evaluation of the optimal site from MASA's machine tool park will be possible. Different simulations will provide the results (function transfers, vibrations ...) and information to select the best machine tool configuration. With this, the machine tool selection stage will be reduced by a $25 \%$.

- Process simulation and set-up: With the implementation of Twin-Control simulations, a comparison between a huge range of different tools and machining conditions can be done, allowing an optimization. In addition, MASA will be able to know before the machining in which operations the chattering risk exists, allowing the modification of machining conditions and the selection of the best alternative option. A reduction of process design and set-up time of around 60\% is expected.

The average process implementation takes currently 43 days. With Twin-Control, it is expected to be reduced to 35 days. This is a reduction of the $20 \%$ in the development of the final process for get a serial production for a part.

\subsection{Scenario of Use 3: Process Control}

The machining process that is designed end set-up to obtain an optimized performance, as presented in scenario of use 2 , is then run in the machine tool by an operator in production conditions.

Under ideal conditions, the operator should only run the process every time a new part is clamped in the fixture. However, different events can take the process from these ideal conditions such as tool wear or breakage, machine tool condition variation and excess/absence of material in raw surfaces.

Apart from this, the operator can usually modify process performance, for example, by modifying the feed rate when process does not go as expected (e.g. chatter occurs).

The application of Twin-Control in this scenario of use leads to a minimization of the impact of the undesirable events during production.

\subsubsection{Local Machine Tool and Process Monitoring and Control Device}

\subsubsection{Implementation and Results}

The monitoring equipment based on the ARTIS Genior Modular and updated in TwinControl will be able to improve process control and facilitate operator's activities. Three new features developed in the project are of special relevance in this scenario. 


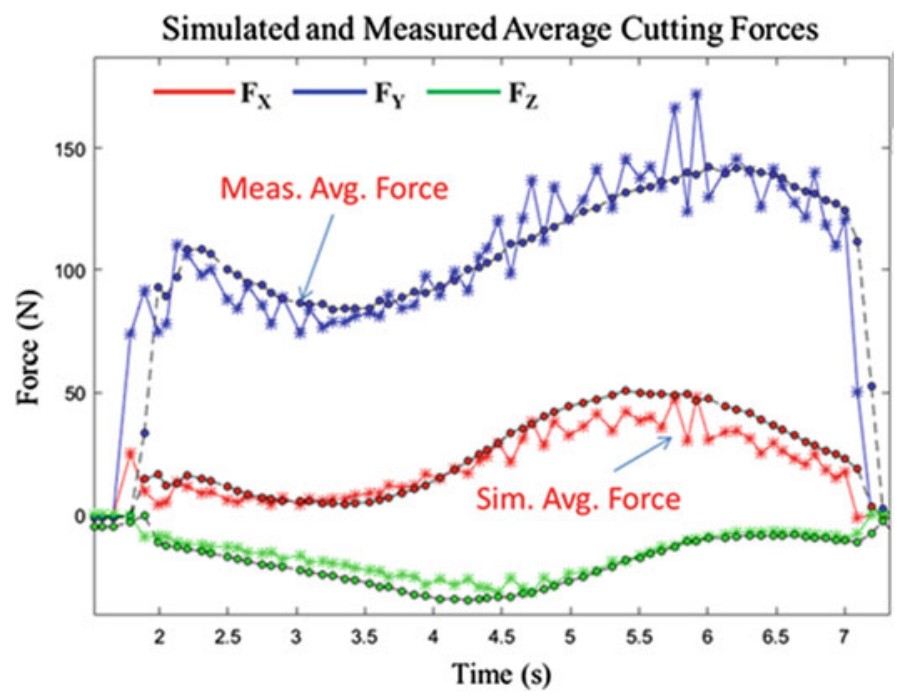

Fig. 15.20 Comparison of simulated and monitored results for the validation operation of the stability roadmap feature of Sect. 2.2 of the book

\subsection{Process Monitoring}

The well-known learning-based process monitoring feature from ARTIS Genior Modular is available at the aerospace use case but does not suit the short to medium batch size faced by MASA.

In this case, the state-of-the-art simulation-based process monitoring approach introduced in Chap. 12 of this book is of special interest. Preliminary results obtained in a Starrag EcoSpeed machine, located at the AMRC (Sheffield, UK), present the possibility to improve process control by embedding process models inside the monitoring hardware, as illustrated in Fig. 15.20.

The estimations done by the process models using real inputs from the machine can be used to determine the ideal conditions and fix thresholds that define anomalous performance of the process.

\subsection{NC Simulation}

The CNC simulation capabilities of ModuleWorks have been integrated in the ARTIS GEM-Visu HMI during Twin-Control project as depicted in Fig. 15.21. An implementation of the aerospace use case has been done by developing a simplified design of the GEPRO 502 machine. The virtual model has been created starting from STL files provided by GEPRO. By using current axis position data recorded by the OPR of the GEPRO 502 machine, the virtual representation of the machine replicates the movements done by the real machine. 


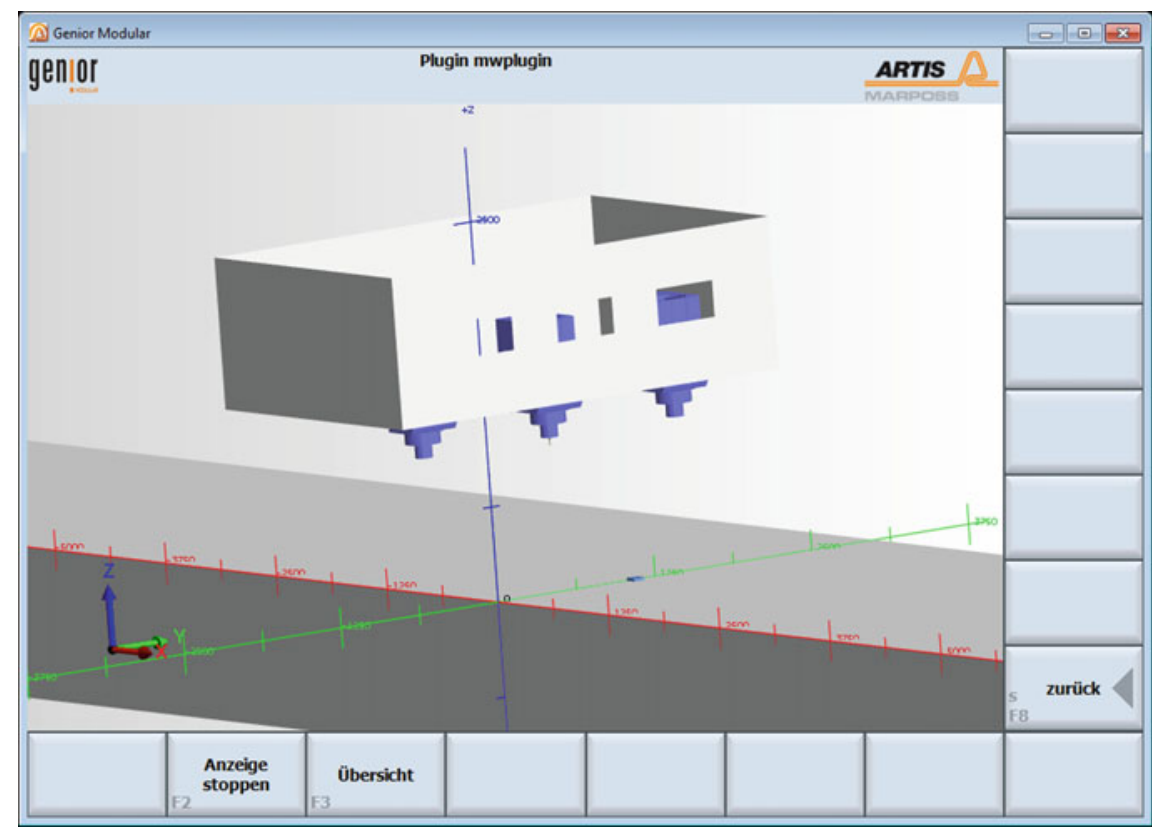

Fig. 15.21 Screenshot of ARTIS HMI showing CNC Simulation feature applied in a simplified GEPRO 502 machine

In machines where the process is not visible for operators, like the GEPRO 502 machine where an opaque curtain blocks the access to the machining region, this feature provides the chance to the operator to control the process.

\subsection{Adaptive Control}

Adaptive feed rate control has been implemented in the GEPRO 502 machine of MASA. This feature consists in the adaptation of feed rate according to spindle consumption and is performed by the ARTIS Genior modular installed in the machine. By applying this feature, when the spindle is under-working, the feed rate is increased; and, when the spindle is overworking, the feed rate is reduced. The objective is to increase productivity by keeping the maximum material removal rate during all the process. To test this, a scalloped sample part, represented in Fig. 15.22, has been used.

First, a learning stage was performed by machining the workpiece longitudinally at a constant feed rate, in this case $4000 \mathrm{~mm} / \mathrm{min}$, depicted in Fig. 15.23. This way, the target spindle consumption value is defined. This value of the spindle consumption will serve as a pattern when the adaptive control is activated.

After that, the adaptive control is activated, and a series of longitudinal machining operations are performed. This time, feed rate is automatically increased in the 
(a)

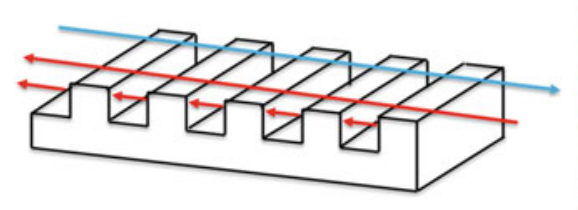

(b)

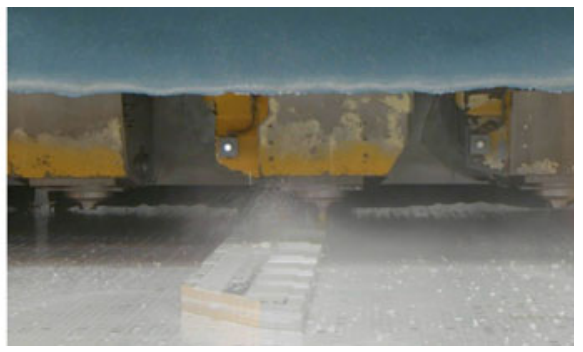

Fig. 15.22 Implementation of adaptive feed rate control in MASA use case: a diagram showing the adaptive control strategy on scalloped part: blue line shows the learning stage; red line shows the adapted stage. $\mathbf{b}$ Picture showing the real workpiece being machine in MASA case

(a)

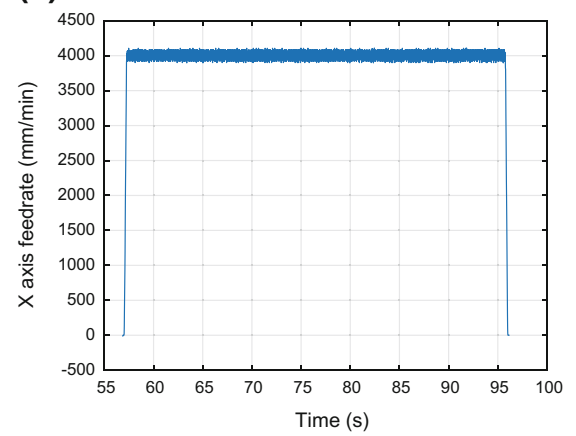

(b)

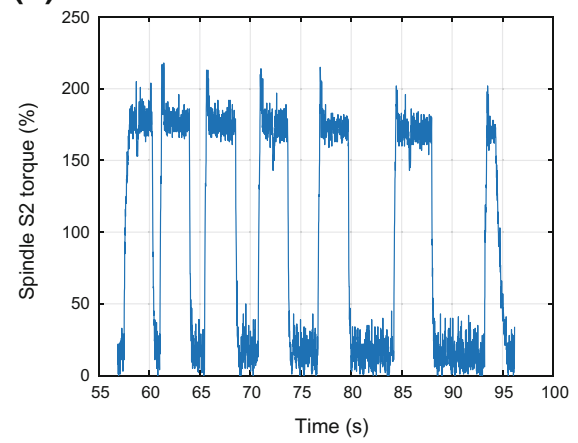

Fig. 15.23 Signals monitored during the learning stage of the adaptive control implementation in MASA use case: a constant feed rate. b Intermittent torque due to scalloped workpiece

regions with no material (Fig. 15.24), trying to reach the learned spindle torque (Fig. 15.23). In these regions, feed rate increases up to $5200 \mathrm{~mm} / \mathrm{min}$, which is the $130 \%$ of the programmed feed rate. This increase (130\%) is a maximum limit fixed for safety reasons. Thereby, shorter machining times were achieved than without the adaptive control.

The differences between the learning stage and the adaptive control stage can be clearly observed in Figs. 15.23a and 15.24a. In the first case, the feed rate reaches values around $4000 \mathrm{~mm} / \mathrm{min}$ and, in the second case, with the adaptive control active, the feed rate reaches values around $5200 \mathrm{~mm} / \mathrm{min}$.

\subsubsection{Impact}

The cycle time of the validation part selected for Twin-Control is approximately $24 \mathrm{~h}$. The application of adaptive feed rate control will provide a reduction of a $10 \%$ in the total cycle time. The long travelling distances, the limitations in cutting conditions 
(a)

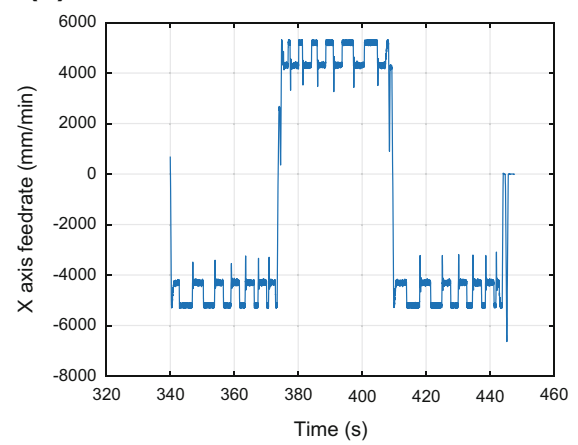

(b)

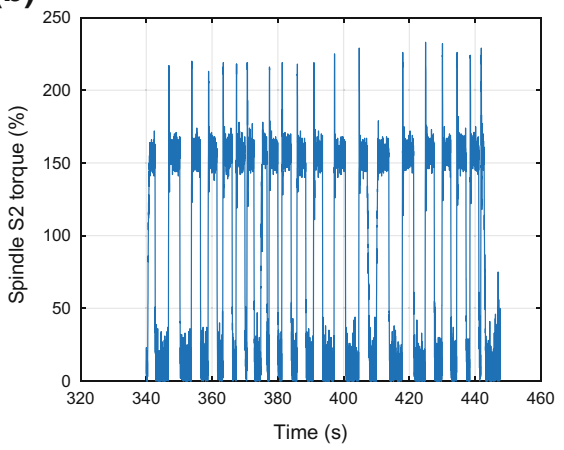

Fig. 15.24 Signals monitored during the adaptive machining in MASA use case: a constant feed rate. b Intermittent torque due to scalloped workpiece

caused by difficult-to-cut materials (e.g. titanium), and the intermittent cutting of typical pocket-type workpieces makes aerospace sector very prone to benefit from adaptive feed rate control.

MASA produces a total of 6000 parts per month, with approximately $1.20 \%$ of scrap parts. This fact, together with the high cost of the scrap parts (average of 600 and $2400 €$ for aluminium and titanium parts, respectively), makes any possibility to increase process reliability very interesting.

Nowadays, the scrap parts due to tool breakage are totally controlled for aluminium machining and no scrap parts are produced by this problem. In case of titanium machining, tool breakage is a problem and a cause of scrap part production. The application of Twin-Control simulation-based process monitoring will allow to predict tool breakage in advance and replace the tool in an early stage.

The average tooling cost for MASA is 6 and $30 €$ per machining hour for aluminium and titanium, respectively. By the application of Twin-Control simulationbased process monitoring, a reduction of the tooling cost of around 5-10\% is expected in titanium machining. In the aluminium machining, it won't have a remarkable effect.

Collisions are quite common in the machines of the aerospace validation scenario, with an average of 20 collisions per year, since an opaque safety curtain is normally blocking the visualization of the machining area and the manufacturing of short batches that require manual set-up operations. The application of CNC simulation capabilities will allow the reduction of collisions in the machines, leading to a reduction of scrap parts and maintenance costs. 


\subsection{Scenario of Use 4: Maintenance}

Preventive maintenance is the most common practice to guarantee machine tools performance in current industrial end-users. Preventive maintenance consists, most of the times, in scheduled actions according to machine usage time. Although this approach reduces machine failures, all the problems cannot be avoided. The proper analysis of the monitored data can help to anticipate to failures.

\subsubsection{Fleet Management System}

The cloud-based fleet-wide platform developed in Twin-Control project capitalizes all the information on all connected machines to support trend analysis of indicators, which is the first step towards predictive maintenance policy. Sections 3.2 and 4.3 of the Twin-Control book provide more insights into the fleet-wide platform developed in Twin-Control project.

\subsubsection{Implementation and Results}

Data from three machines located at MASA is uploaded to the cloud platform for machine tools hosted on a dedicated server (https://twincontrol.kasem.fr/). As machine operating data is one main input of the platform, reliability of machine tool connection is important. Every night, for 1 year and a half, behaviours indicators are extracted from raw data, operating and characterization test reports are generated.

MASA production cycle time is long, and parts are not produced in series. It means that to have comparable indicators between parts specific moves should be identified or requested, as done in the characterization tests. Additionally, laws between move indicator and for instance move length and distance can be characterized.

\subsection{Y-Axis Power Consumption Drift}

Analysis of power consumption of each $Y$-axis linear move according to speed and length shows a gap in the indicators from the 2017/06/22 when data transfer between machine and fleet platform is restored after few days, as depicted in Fig. 15.25. A maintenance activity report from MASA shows that during the week of the 2017/06/30 a problem with a chip accumulation in the $Y$-axis occurred, causing several components damage and replacement as shown in Fig. 15.26.

In this scenario, data transfer has been lost just before the drift of power. It can be assumed that the drift will be detected more than 3 days before. 


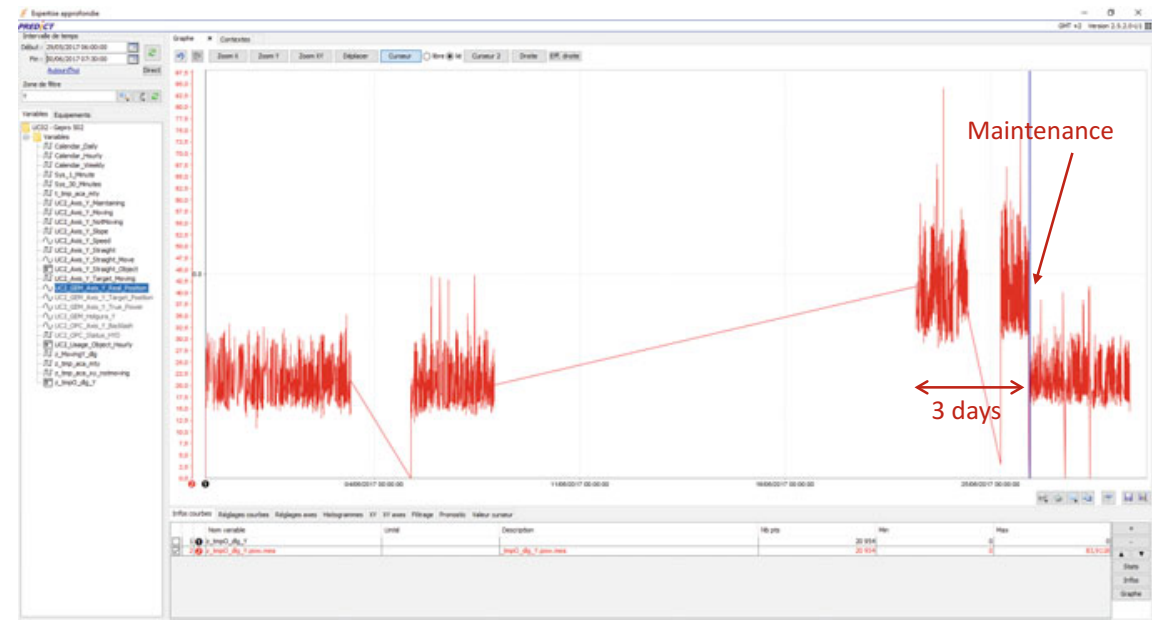

Fig. 15.25 Identification of anomalous performance of the $Y$-axis of GEPRO 502 machine using power-based indicators

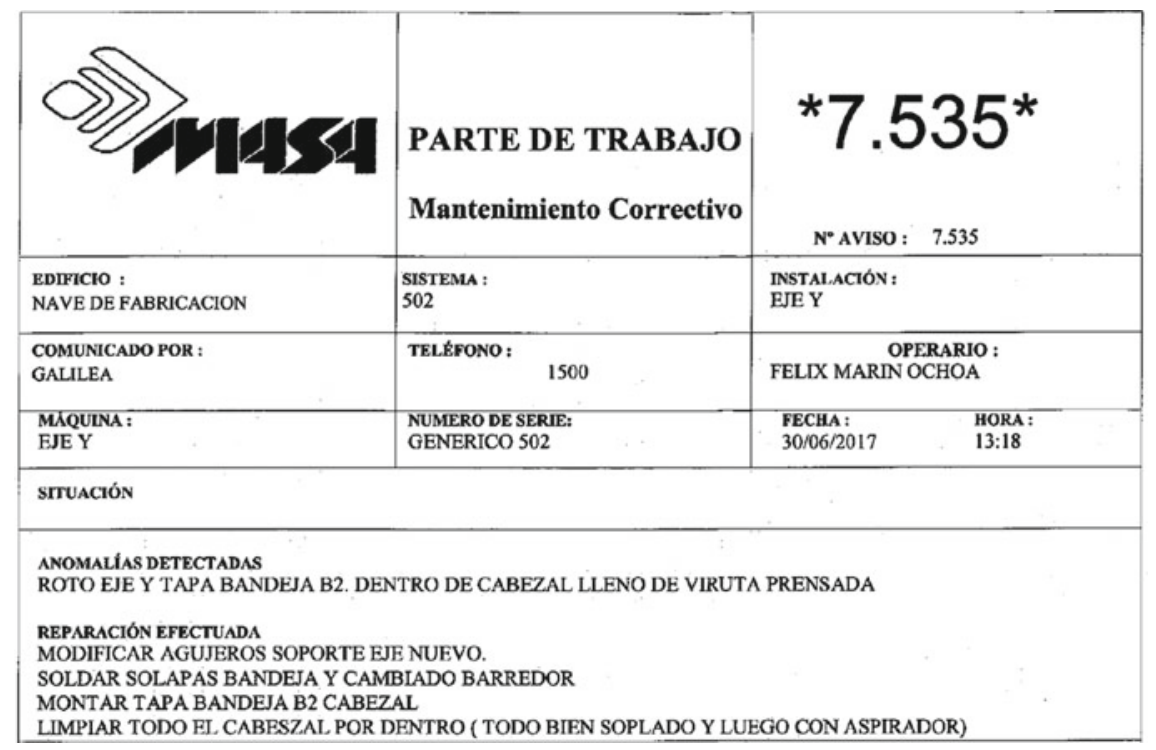

Fig. 15.26 Extract of MASA maintenance report from 2017/06 (in Spanish), indicating a maintenance action due to chip accumulation in $Y$-axis

\subsection{Characterization Tests}

Around 30 characterization tests have been made and analysed during the project. Due to its importance for MASA, this analysis is focused in the backlash indicator 


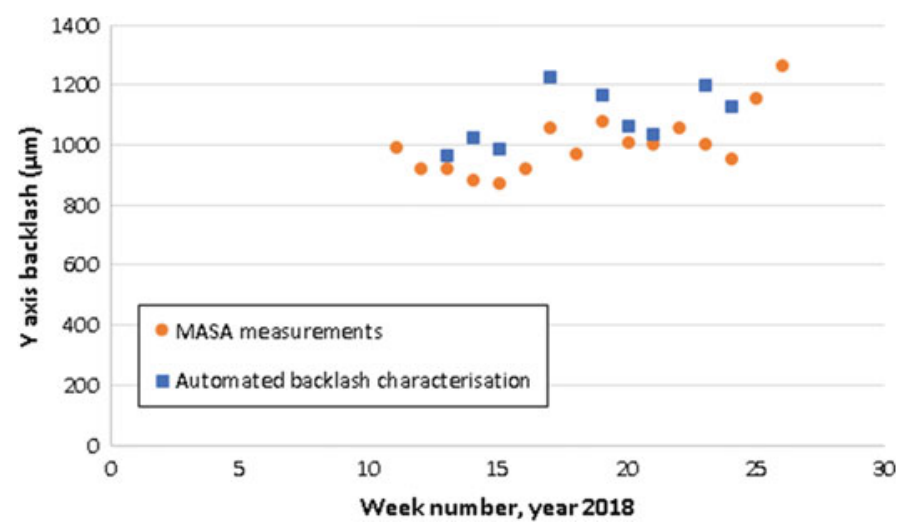

Fig. 15.27 Comparison of the $Y$-axis backlash obtained from circularity tests and through direct measurement

for the $Y$-axis, which is computed from the circularity tests. The trend of the $Y$-axis backlash calculated thanks to the characterization test is presented in Fig. 15.27, where the experimental measurements done by MASA are also plotted. It can be observed that the automated test is able to detect the same trend and provide good quantitative estimation of the backlash.

\subsubsection{Impact}

As mentioned before, MASA maintenance activities are based on preventive strategy. Periodic check of the most critical systems (axes backlash, hydraulic system and electrical components...) is performed by maintenance experts. This check supposes machine downtime and does not avoid machine failure.

With the application of a predictive maintenance strategy, MASA will be able to minimize the possibilities to suffer the failure. Thank to early detection of a drift in the machine behaviour, maintenance actions can be optimized, minimizing machine downtime. In addition, part of preventive actions could be eliminated, reducing overall maintenance costs and machine downtime.

Backlash of feed drives is one of the most critical aspects to get scrap parts in MASA. Currently, MASA checks the backlash manually, which is time-consuming. The implementation of MT characterization tests, where backlash is automatically determined makes easier the control of this parameter and minimizes the risk of getting a scrap part due to axis backlash. It is expected a reduction of the $10 \%$ in scrap parts due to this implementation.

Proactive events also contribute to the reduction of energy consumption of the machine because in most cases abnormal behaviours an equipment will increase its consumption with factors up to 3 as observed in MASA cases. 


\subsection{Conclusions}

This chapter presents the implementation and obtained results of the technical developments done in Twin-Control project in the proposed aerospace industrial validation scenario. A specific approach for Twin-Control evaluation is defined, based on different scenarios of use defined in the project. The results and the impact of Twin-Control features are structured according to these scenarios of use.

Twin-Control shows a set of features that can help aerospace machine tool builders and users to improve their processes. A summary of the impact caused by the application of Twin-Control is listed next:

- A reduction of a $10 \%$ in the total development time of a new customized machine tool.

- A reduction of the $20 \%$ in the design and set-up of a new process.

- A reduction of $10 \%$ in cycle time.

- A reduction of tooling cost of 5-10\%, depending on the workpiece.

- Reduction of machine downtime and maintenance costs thanks to predictive maintenance approach.

- A reduction of scrap parts of the $10 \%$.

Open Access This chapter is licensed under the terms of the Creative Commons Attribution 4.0 International License (http://creativecommons.org/licenses/by/4.0/), which permits use, sharing, adaptation, distribution and reproduction in any medium or format, as long as you give appropriate credit to the original author(s) and the source, provide a link to the Creative Commons license and indicate if changes were made.

The images or other third party material in this chapter are included in the chapter's Creative Commons license, unless indicated otherwise in a credit line to the material. If material is not included in the chapter's Creative Commons license and your intended use is not permitted by statutory regulation or exceeds the permitted use, you will need to obtain permission directly from the copyright holder. 